\title{
Emerging signs of strong reciprocity in human ontogeny
}

\section{Erin Robbins* and Philippe Rochat}

Department of Psychology, Emory University, Atlanta, GA, USA

\section{Edited by:}

Steven E. Mock, University of

Waterloo, Canada

Reviewed by:

David Liu, University of California San Diego, USA

Jeff Loucks, University of

Washington, USA

\section{${ }^{*}$ Correspondence:}

Erin Robbins, Emory Infant and Child

Lab, Department of Psychology,

Emory University, 36 Eagle Row,

Atlanta, GA 30322, USA.

e-mail: eerobbi@emory.edu

\begin{abstract}
Strong reciprocity is considered here as the propensity to sacrifice resources to be kind or to punish in response to prior acts, a behavior not simply reducible to self-interest and a likely force behind human cooperation and sociality. The aim was to capture emerging signs of strong reciprocity in human ontogeny and across highly contrasted cultures. Three- and 5 -year-old middle class American children $(N=162)$ were tested in a simple, multiple round, three-way sharing game involving the child, a generous puppet, and a stingy puppet. At the end of the game, the child was offered an opportunity to sacrifice some of her personal gains to punish one of the puppets. By 3 years, American children demonstrate a willingness to engage in costly punishment. However, only 5-year-olds show some evidence of strong reciprocity by orienting their punishment systematically toward the stingy puppet. Further analyses and three additional control conditions demonstrate that such propensity is not simply reducible to (a) straight imitation, or (b) inequity aversion. To assess the relative universality of such development, a group of 5- to 6-year-old children from rural Samoa $(N=14)$ were tested and compared to age and gender-matched American children. Samoan children did not manifest the same propensity toward strong reciprocity. The results are interpreted as pointing to (1) the developmental emergence of an ethical stance between 3 and 5 years of age, and (2) that the expression of such stance by young children could depend on culture.
\end{abstract}

Keywords: strong reciprocity, costly punishment, cross-cultural psychology, sharing, egalitarianism, moral reasoning

\section{INTRODUCTION}

Defectors of potential cooperation tend to be punished, even in anonymous interactions and even when such punishment is costly, not rational in either long or short economic terms, with the caveat that it can vary across cultures (Henrich et al., 2006; Henrich and Henrich, 2007). Strong reciprocity captures the propensity to sacrifice resources, either to reward or to punish, in response to perceived kindness or un-kindness (Fehr et al., 2002). The question of interest here is when does such propensity start to emerge in human ontogeny and what is its significance in child development.

Recent evidence suggests that human pro-social tendencies are deeply rooted in development, already present from the middle of the first year of life, as in the case of infants detecting pro-social versus anti-social acts perpetrated by third-party protagonists (Kuhlmeier et al., 2003; Hamlin et al., 2007). By their second birthday, children manifest the explicit inclination to help and collaborate with others (Warneken et al., 2007; Tomassello, 2008), as well as to display empathic responses toward suffering others (Zahn-Waxler et al., 1992; Eisenberg and Fabes, 1998). It is also by the second year of life that children begin to show explicit attention to social norms (Kagan, 1988).

Drawing on an analogy with existing theories on human language (Pinker, 2008), some theories postulate the existence of a universal and innate moral instinct of the species, a moral grammar (Hauser, 2006) unique to humans and deeply rooted in the evolution of particular emotional responses (Haidt and Joseph, 2004). However, the analogy with language remains problematic (Dupoux and Jacob, 2007), particularly in view of children's slow developing sense of fairness or egalitarianism (i.e., inequity aversion, Fehr et al., 2008) and moral reasoning (Piaget, 1932; Kohlberg, 1981), both showing significant variations across cultures (Snarey, 1985; Rochat et al., 2009).

By 21 months children are explicit about possession. Personal pronouns and adjectives like "mine!" become part of the child's vocabulary as an index of explicit ownership over things, opening up possibilities for sharing, negotiation, and bartering with others (Faigenbaum, 2005). Research documents an important developmental progression in the sharing of rewards and resources. Three-year-olds demonstrate marked self-maximizing (selfish) and self-interested tendencies when asked to share, as in the example of distributive justice games (Lane and Coon, 1972; Hook, 1978). Cross-cultural studies show that self-maximizing tendencies by 3 -year-olds tend to be moderated by the social and physical environment of the child. Self-interest is significantly attenuated in 3 -year-olds growing up in rural, more communal small-scale traditional societies of Fiji and Peru when compared to middle class children from urban and industrial Brazil, China, or United States (Rochat et al., 2009). However, despite these variations, it appears that regardless of culture, there is a decrease in self-maximizing tendencies in sharing between 3 and 5 year of age (Rochat et al., 2009). By 5 years, children are reported to apply a strict equity or egalitarianism principle to their distributions. Sharing and distributive justice are presumed to develop with the child's ability to reason in terms of proportion (Inhelder and Piaget, 1964), 
eventually leading them to apply principles of proportionality by factoring relative work, effort, production, need, or starting wealth from around 7 years of age (Damon, 1975).

At an age when children might still be overwhelmingly driven by self-maximizing tendencies, they also appear capable of generating social preferences and value judgments about others on the basis of how they give. From 4 years of age, children base social judgments on absolute quantities of a distributed good, for example judging one of two puppets as "nicer" because it gives them more valuable chips (McCrink et al., 2009). By 5 years, children begin to show signs that they judge the relative niceness of the puppet based on the proportion of the chips the puppet is willing to sacrifice, in the same way that adults tend to judge whether a resource distribution is more or less equitable based on proportional reasoning (McCrink et al., 2009).

Recent evidence also suggests that by 3.5 years of age, even when they are not themselves recipient of a distribution, children demonstrate selective preference in their distribution of resources based on past relations (Olson and Spelke, 2008). In their study, Olson and Spelke asked 3- to 4-year-olds to help a doll distribute resources to other dolls that were described as either close relations (in-group) or strangers (out-group); dolls that had or had not previously shared with the distributing doll (direct reciprocity); or dolls that did or did not previously share with other dolls (indirect reciprocity). In this situation children demonstrated tendencies suggestive of an early sensitivity to close relations as well as direct reciprocity (i.e., tit-for-tat exchanges) and indirect reciprocity (long term pay-off/reputation based exchanges). Note that all this is demonstrated even though the sharing game involves puppet dolls and not real protagonists.

Although tendencies toward direct and indirect reciprocity are both considered as foundational aspects of cooperation and appear to "extend deep in human development" (Olson and Spelke, 2008), it is not clear yet how such tendencies eventually develop to become strong reciprocity, which represents a marked qualitative shift in meaning. Strong reciprocity, as indexed by costly punishment, entails the sacrifice material resources to punish another individual and is considered here as the expression of principled decisions by the child. Engaging in costly punishment therefore requires the child to forego personal gain to enforce a norm (e.g., for fairness) that has been violated. In the willingness to sacrificing her own resources, the child demonstrates an ethical (principled) stance toward others that is qualitatively different and presumably more advanced than simple reciprocity (e.g., tit-for-tat reciprocation). In addition, the foundational aspect of strong reciprocity needs to be specified. For example, is it based on the adoption of some moral principle (what we call here an ethical stance), or might it rest only on an early propensity toward inequity aversion as proposed by Fehr et al. (2008)? Furthermore, little is known regarding the influence of culture on the development leading children from a reciprocity that might be direct or indirect to one that is strong, i.e., principled and presumably independent of future self-maximizing rewards.

In this context, the question we asked is twofold: First, what are the ontogenetic origins of strong reciprocity and second, what might be the potential influence of culture in the expression of such a tendency in children's development?
As a general working hypothesis, we expected that strong reciprocity (as expressed in selective costly punishment) would develop in the preschool years, becoming evident by 5-6years when children are also documented as becoming proficient and complex readers of others' minds (Wellman and Liu, 2004), a development that is predictable across cultures (Callaghan et al., 2005).

In a series of studies, we present evidence that first signs of strong reciprocity emerge by 5 years. However, we also provide some evidence that contrary to our expectations, the expression of strong reciprocity by 5- to 6-year-olds can vary depending on culture (i.e., affluent, urban United States versus rural, traditional, and group-oriented Samoa, see Study 6).

\section{GENERAL METHODOLOGY}

In six different studies, children participated in a triadic (threeway), multi-round sharing game in which they were asked to split coins (poker chips of uniform size, shape, and color) between themselves and two protagonists. To provide an incentive for playing, prior to the onset of the game children visited a makeshift toy store where the experimenter explained how accumulated coins could be used to purchase various prizes (e.g., small toys and stickers $<\$ 1$ USD). Children were also informed that their sharing partners could similarly spend their winnings. To ensure that children had associated the coins with the ability to win prizes, at several points throughout the game children were prompted to explain how the coins worked.

Study 1 examined children's sense of reciprocity by asking participants to share coins with stingy and generous partners. Study 2 replicated the task and then expanded this methodology to include a more direct test of strong reciprocity, a costly punishment task in which children could sacrifice material gains to punish one (or both) of the sharing partners. Three controls (Studies 3-5) assessed whether children were sensitive to the actions of the stingy or generous puppets. Finally, based on the same procedure, a comparison with a group of same age Samoan children in Study 6 offered the opportunity to examine potential cross-cultural variations in the emerging expression of strong reciprocity (selective costly punishment).

\section{BASELINE ASSESSMENT}

In order to assess children's general sense of equitable and proportional sharing, a pre-test was performed first. Children were asked to split nine coins between three identical toy ducks. We limited the number of items shared to nine to avoid an exclusive reliance on counting, which was presumably not yet in the register of the younger children.

For the pre-test trial and the four sharing rounds that followed (see description of the triadic sharing game below), an unfamiliar female Experimenter sat at a table opposite the child and arranged the valuables to be split (poker chips) in a circular formation on the center of the table before instructing" children to "split the coins" (Figure 1). In the pre-test and in the triadic sharing game that followed, children indicated ownership of the coins by placing

\footnotetext{
1"Split" was used in place of other instructions (e.g. "share" or "divide") which are often connotative of parsing a given object equitably, or into equal parts.
} 


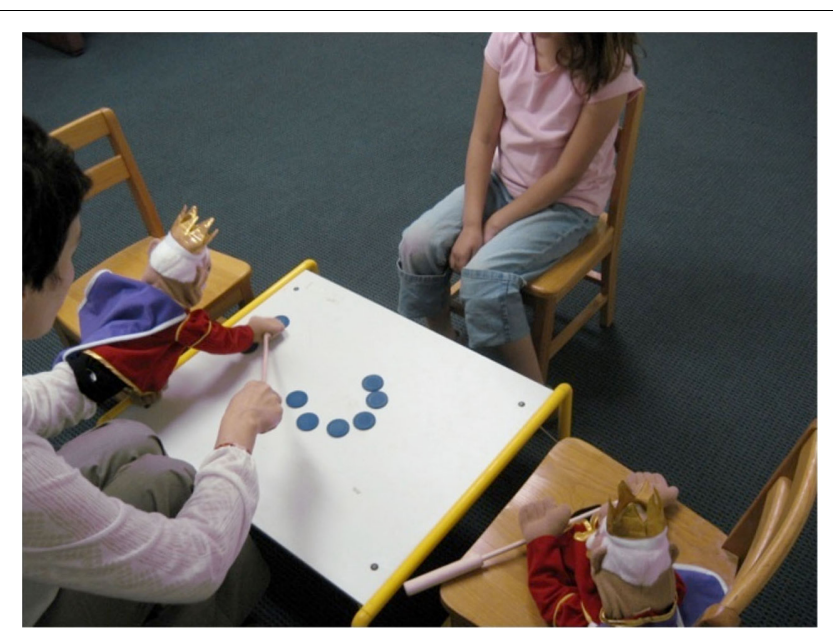

FIGURE 1 | Experimental situation in the United States. Identical puppets were animated in turn by the Experimenter during the three-way sharing game (here the "stingy" puppet maximizes coins for himself).

them on the table in front of the intended recipient. After the child confirmed this distribution, the winnings were deposited into banks (transparent plastic containers, each belonging to one of the sharing protagonists) for safekeeping.

\section{TRIADIC SHARING GAME}

Following baseline pre-test, the child was tested in a triadic (threeway) sharing game using nine pretend, yet valuable coins (poker chips). In four successive trials, the child was asked to split the coins in turn with two puppets that acted either with marked generosity or marked stinginess (Studies 1, 2, and 5). In three control conditions, children participated in the same triadic share task, but with a slight modification. In Study 3 (Generous Control) children interacted with two generous puppets. In Study 4 (Stingy Control) children interacted with two stingy puppets. Finally, in Study 5 (Non-agentive Control), children interacted with two passive puppets. In this latter control condition, children are presented with a pre-established distribution without seeing the puppets actively sharing either stingily or generously.

The two identical, high-quality plush hand-puppets $(43 \mathrm{~cm}$ tall) dressed as kings sat at the table to the right and left of the child and were animated by the Experimenter with the same vocalizations and mannerisms throughout the experiment (Figure 1). In four successive rounds, the two puppets always started, one sharing generously (four coins to the other puppet, four coins to the child, and only one to itself), and one sharing stingily (keeping seven coins for itself, giving one to the other puppet, and only one to the child). By sharing last, children observed the maximum effect of the puppets' generous or stingy behaviors.

In all studies, when it was the child's turn, we recorded and analyzed as a dependent measure the total number of coins distributed by the child to herself and to the two puppets as a function of the four sharing rounds.

Researchers counterbalanced both the position of the characters (i.e., the location of the generous and stingy puppet relative to the child) as well as which puppet shared first to avoid the potential of a side bias or order effect. Children were clearly and unambiguously informed that each protagonist would keep and could accumulate received coins to purchase attractive toys at a makeshift toy store they visited prior to testing. To re-emphasize the accumulative nature of the game, at the end of each round the Experimenter asked the child to compare her bank with the banks of the two puppets and determine which player had accumulated the most coins.

\section{SELECTIVE COSTLY PUNISHMENT TEST}

In Studies 2-6, at the conclusion of the triadic sharing game children were probed for signs of strong reciprocity in a final costly punishment test. After the last round of sharing, the Experimenter instructed children to examine the contents of the three banks and determine which player had the most coins. Next, the Experimenter asked whether the outcome of the game was fair. It was then proposed that as the "banker" of the game, the Experimenter could take five coins away from a puppet of the child's choosing, but only if the child gave up one of her own coins. To underscore the costly nature of this decision, the Experimenter reminded children that coins sacrificed by the child or taken from the puppets could not be used in the store. The Experimenter then asked if the child would like to proceed. A child who responded "yes" had to give the Experimenter one of her own coins before identifying the puppet that should be punished ${ }^{2}$. The Experimenter offered a chance for the child to engage in costly punishment again by asking if she would like to sacrifice another coin (i.e., "Would you like to give me another coin and I can take five coins away from one of the kings?"). This procedure repeated until the child declined to offer a coin, or until one of the banks was emptied. For the dependent measure, we recorded the number of coins sacrificed to punish each puppet.

\section{PARTICIPANTS}

Except where noted in Study 6 (cross-cultural comparison), we sampled children from predominantly middle- to upper-middle class families living in metro-Atlanta, GA, USA (pop: $>5$ million). In accordance with our IRB-approved protocol, children were enrolled into the study through the consent of parents, who were invited by phone and e-mail to participate. Half of the participants completed the study at a university research lab. We recruited other children from small after-school programs and pre-schools less than $3 \mathrm{~km}$ from the university campus. When appropriate, we obtained additional consent from teachers and school administrators. Table 1 provides descriptive statistics of the participants in Studies 1-6.

\section{STUDY 1 (CHILD-STINGY-GENEROUS TRIADIC SHARE WITHOUT COSTLY PUNISHMENT) HYPOTHESES}

On the basis of prior studies that have found age-related trends in egalitarian sharing behavior (Fehr et al., 2008; Rochat et al., 2009), we hypothesized that 3- and 5-year-olds would differ in

${ }^{2}$ The word "punishment" was not used to explain this task to participants in an effort to avoid biasing children toward participating. 
Table 1 | Descriptive statistics of participants in Studies 1-6.

\begin{tabular}{|c|c|c|c|}
\hline & Males $(N)$ & Females $(N)$ & $\begin{array}{l}\text { Mean age } \pm S D \\
\text { (in months) }\end{array}$ \\
\hline \multicolumn{4}{|c|}{ Study 1 (Child-Stingy-Generous Triadic Share without } \\
\hline \multicolumn{4}{|c|}{ Costly Punishment), $N=36^{a}$} \\
\hline 3 -year-olds $(N=18)$ & 10 & 8 & $44.27 \pm 4.85$ \\
\hline 5 -year-olds $(N=18)$ & 11 & 7 & $67.33 \pm 5.85$ \\
\hline \multicolumn{4}{|c|}{ Study 2 (Child-Stingy-Generous Triadic Share and Costly } \\
\hline \multicolumn{4}{|l|}{ Punishment), $N=66$} \\
\hline 5 -year-olds $(N=33)$ & 18 & 15 & $64.15 \pm 4.90$ \\
\hline \multicolumn{4}{|c|}{ Study 3 (Generous Control with Triadic Share and Costly } \\
\hline \multicolumn{4}{|l|}{ Punishment),$N=24$} \\
\hline 3 -year-olds $(N=12)$ & 4 & 8 & $44.25 \pm 6.03$ \\
\hline 5 -year-olds $(N=12)$ & 4 & 8 & $65.89 \pm 7.68$ \\
\hline \multicolumn{4}{|c|}{ Study 4 (Stingy Control with Triadic Share and Costly Punishment), } \\
\hline $\begin{array}{l}\mathbf{N}=\mathbf{2 4} \\
\text { 3-year-olds }(N=12)\end{array}$ & 7 & 5 & $45.00 \pm 4.35$ \\
\hline 5 -year-olds $(N=12)$ & 5 & 7 & $60.92 \pm 5.90$ \\
\hline \multicolumn{4}{|c|}{ Study 5 (Non-Agentive Control with Triadic Share and Costly } \\
\hline $\begin{array}{l}\text { Punishment), } \boldsymbol{N = 1} \\
\text { 5-year-olds }(N=18)\end{array}$ & 9 & 9 & $64.17 \pm 3.62$ \\
\hline \multicolumn{4}{|c|}{ Study 6 (Cross-Cultural Comparison of us and Samoan 5 -Year-Old } \\
\hline \multicolumn{4}{|l|}{ Children), $N=28^{c}$} \\
\hline Samoa $(N=14)$ & 7 & 7 & $68.30 \pm 5.83$ \\
\hline US $(N=14)$ & 7 & 7 & $65.18 \pm 5.75$ \\
\hline
\end{tabular}

For 3-year-olds in all studies, participants ranged between 34 and 50 months and 5-year-olds ranged between 58 and 70 months. ${ }^{a}$ Study 1: two participants were omitted on the basis that they did not complete the study, yielding an attrition rate of $6 \%$. ${ }^{b} T 0$ control for the possibility that inequity aversion was driving the selective costly punishment results amongst older children, Study 5 sampled only 5 -year-olds. 'The US children included in Study 6 were randomly selected from the sample of 5-year-olds who participated in Study 2.

their distribution of the coins, particularly with regard to the stingy and generous puppets. As for rationale, we considered that if reciprocity guides children's sharing, they should give more coins to the generous rather than the stingy puppet (who violates an expectation of fairness) when it is their turn to split. We hypothesized that this tendency should become more marked as a function of the four sharing rounds as children observe the consistency of the generous and stingy puppets' actions, and that this expression of reciprocity should be evident by 5 years of age and not earlier.

\section{RESULTS}

We analyzed the number of coins (out of nine) distributed to each protagonist as the dependent measure in a 4 (round) $\times 3$ (recipient) $\times 2$ (age) $\times 2$ (gender) mixed-design ANOVA. Children's performance on the three-way sharing pre-test, coded as a binomial variable indicating whether or not children split coins equally (3-3-3) between the three dolls, was included as covariate to control for potential differences in children's abilities to reason proportionally. Neither gender nor pre-test performances were found to be significant factors, $F_{1,31}=1.57$ and 0.622 , respectively.

Analyses yielded a three-way interaction of round, recipient, and age, $F_{6,29}=2.57, p=0.040, \eta^{2}=0.080$. Taking into consideration only the child as recipient, in a follow-up test to assess children's self-maximizing tendencies over the course of the sharing game we observed a significant main effect of round, $F_{3,32}=3.41, p=0.029, \eta^{2}=0.179$. Overall, as a function of round, children of both ages tended to accumulate more coins for themselves. However, note that there was a marginally significant interaction of round by age $\left(F_{3,32}=2.44, p=0.082\right.$, $\left.\eta^{2}=0.078\right)$ suggesting a stronger trend for younger children. The mean difference in the number of coins distributed to the child between Rounds 1 and 4 was greater for 3-year-olds $(\mathrm{MD} \pm \mathrm{SE}=2.66 \pm 0.918, p<0.01$ based on Bonferroni-adjusted comparisons) and not statistically significant for 5-year-olds $(\mathrm{MD} \pm \mathrm{SD}=0.301 \pm 0.664)$.

Additional follow-up tests assessed children's treatment of the two puppets across the four rounds of sharing. Amongst 3year-olds we observed no significant main effects or interactions of round and recipient. On average, these children distributed roughly the same number of coins to the stingy $(M \pm \mathrm{SD}=$ $2.05 \pm 1.60)$ and generous $(M \pm \mathrm{SE}=1.98 \pm 1.76)$ puppet in each round (see Figure 2, left panel). Therefore, 3-year-olds did not express any selective discrimination between the two protagonists. In contrast, 5-year-olds increasingly discriminated between stingy and generous characters as a function of rounds (see Figure 2, right panel). There was a significant interaction of round by recipient for 5-year-olds $\left(F_{3,15}=2.91\right.$, $\left.p=0.037, \eta^{2}=0.084\right)$. Although the mean difference in the number of coins given to the generous and stingy was not significant in Round $1(\mathrm{MD} \pm \mathrm{SE}=0.048 \pm 0.387)$, children significantly favored the generous over the stingy puppet in Round $2(\mathrm{MD} \pm \mathrm{SE}=0.667 \pm 0.226)$, Round $3(\mathrm{MD} \pm \mathrm{SE}=$ $0.952 \pm 0.337)$, and Round $4(\mathrm{MD} \pm \mathrm{SE}=1.10 \pm 0.388)$, all $p<0.05$ based on pairwise comparisons, Bonferroni-adjusted).

Together, the results of the triadic sharing game indicated that sensitivity to the relative generosity or stinginess of the sharing partners, as a function of round, is evident by 5-year-olds only. Five-year-old children in our sample maximized their gains (although to a lesser extent than their 3-year-old counterparts) while simultaneously becoming more discriminating in their distributions to the stingy over the generous puppet. These findings indicate that 5- but not 3-year-olds deal with sharing partners depending on their character (generous or not) in acts of reciprocity and in response to acts that defy norms of fairness. However, without evidence of self-sacrifice and costly punishment on the part of the child, it is difficult to determine whether children are able to by-pass mere reciprocity to also show signs of strong reciprocity. The next study was devised to test for this possibility.

\section{STUDY 2 (CHILD-STINGY-GENEROUS TRIADIC SHARE WITH COSTLY PUNISHMENT)}

In a more direct test of strong reciprocity, we repeated with 66 children (33 three-year-olds and 33 five-year-olds, see Table 1) the Triadic Sharing game described in the first study, adding a followup condition (the Costly Punishment task) to determine whether children would be willing to sacrifice some of their own accumulated coins to punish one of the two protagonists, particularly the stingy, unfair puppet. 


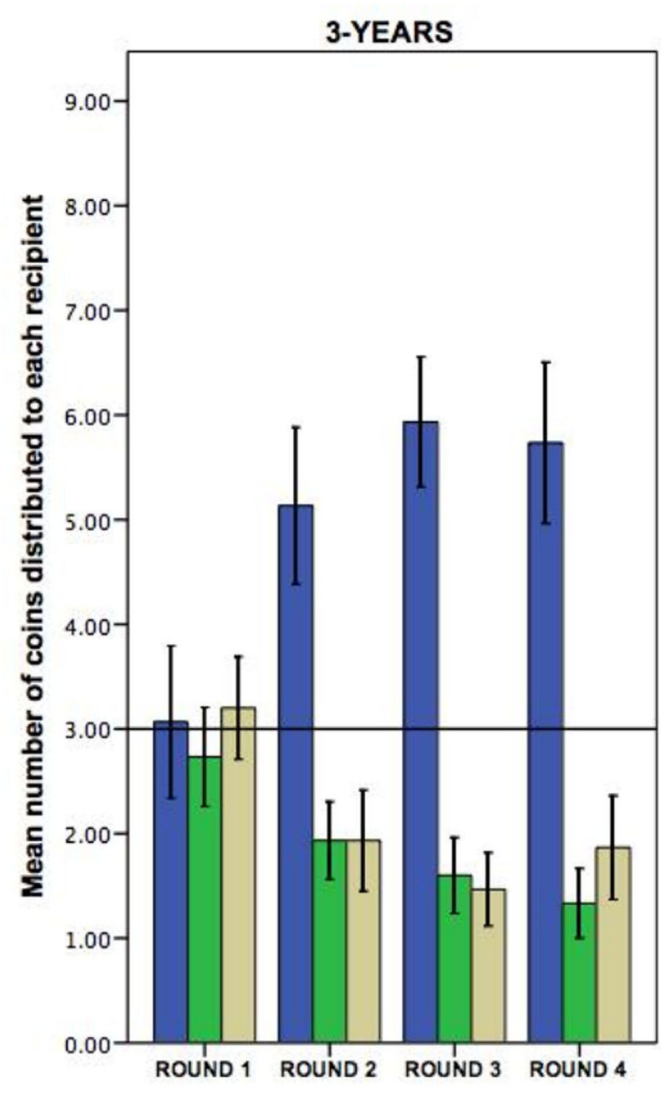

FIGURE 2 | Children's distributions in triadic sharing in Study 1.

Vertical axis represents mean number of coins (maximum nine)

distributed by 3-year-olds (left panel) and 5-year-olds (right panel) to each

\section{HYPOTHESES}

We expected to replicate the findings in Study 1 regarding the four rounds of three-way sharing with the stingy and generous puppets. We predicted that 5-year-olds would show reciprocity compared to 3-year-olds who should self-maximize more and be less selective regarding the stingy or generous character of the puppets. In relation to strong reciprocity, in the additional costly punishment task we expected 5-year-olds to engage in costly sacrifice to punish the stingy protagonist when offered the opportunity, going above and beyond reciprocity, and thus starting to take a principled ethical stance (see introduction for developmental rationale).

\section{RESULTS}

As in Study 1, we analyzed the number of coins (out of 9) distributed to each player as the dependent measure in a 4 (round) $\times 3$ $($ recipient $) \times 2$ (age) $\times 2$ (gender) mixed-design ANOVA. Neither gender $\left(F_{1,61}=0.628\right)$ nor children's performance on the threeway pre-test, included here as a covariate $\left(F_{1,61}=0.446\right)$, were found to be significant factors.

Results yielded a significant three-way interaction of round, recipient, and age, $F_{6,59}=3.73, p<0.01, \eta^{2}=0.531$. In a follow-up test to assess children's degree of self-maximizing and considering only what children gave to themselves, we observed a main effect of round, $F_{3,62}=7.57, p<0.01, \eta^{2}=0.544$. On

\section{5-YEARS}

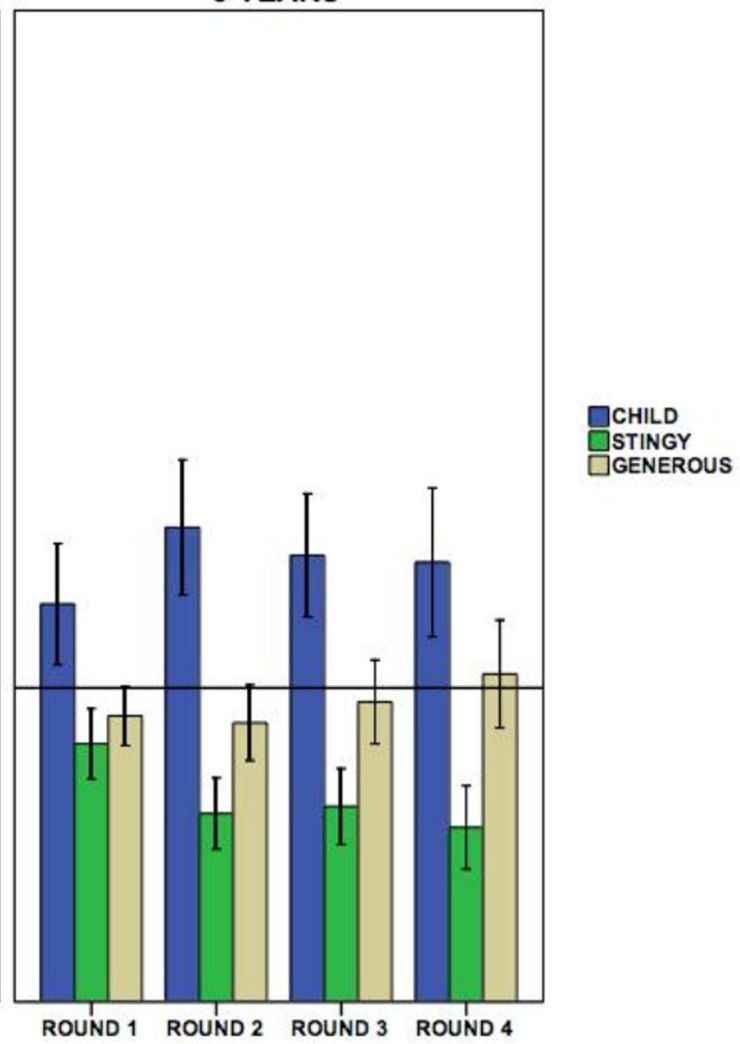

protagonist (child, stingy, and generous) as a function of round (1-4). The horizontal line represents absolute equity (three coins). Bars represent $\pm 1 \mathrm{SE}$. average, both 3- and 5-year-olds increased the number of coins given to themselves over the course of the game, from Round 1 $(M \pm \mathrm{SD}=3.97 \pm 2.11)$ to Round $4(M \pm \mathrm{SD}=4.91 \pm 2.26)$.

With regard to their treatment of the two puppets, over the four sharing rounds we found no significant trends for 3year-olds. These younger children did not differentiate significantly between the stingy $(M \pm \mathrm{SD}=2.24 \pm 1.50)$ and generous $(M \pm \mathrm{SD}=2.20 \pm 1.58)$ puppets at any point in the game (Figure 3, left panel). In contrast, as in Study 1, 5-year-olds discriminated between the puppets. For these children, a significant interaction of round and recipient $\left(F_{3,20}=5.65, p<0.01\right.$, $\left.\eta^{2}=0.361\right)$ points to a preferential treatment of the generous over the stingy protagonist. As predicted, the mean difference in the number of coins distributed to the generous and stingy puppet was significant in Round $2(\mathrm{MD} \pm \mathrm{SE}=0.635 \pm 0.260)$, Round $3(\mathrm{MD} \pm \mathrm{SE}=0.458 \pm 0.254)$, and Round $4(\mathrm{MD} \pm \mathrm{SE}=0.667 \pm$ 0.278 ), all $p<0.01$ based on Bonferroni-adjusted pairwise comparisons. This analysis of the recipient by round interaction suggests that as the game progressed, 5-year-olds gave more to themselves at the expense of the stingy rather than the generous puppet. The disparity between puppets (particularly in Rounds 2 4, see Figure 3, right panel) is likely a result of the child penalizing the stingy protagonist by giving more to herself rather than the child sacrificing her own coins to give more to the generous puppet. 


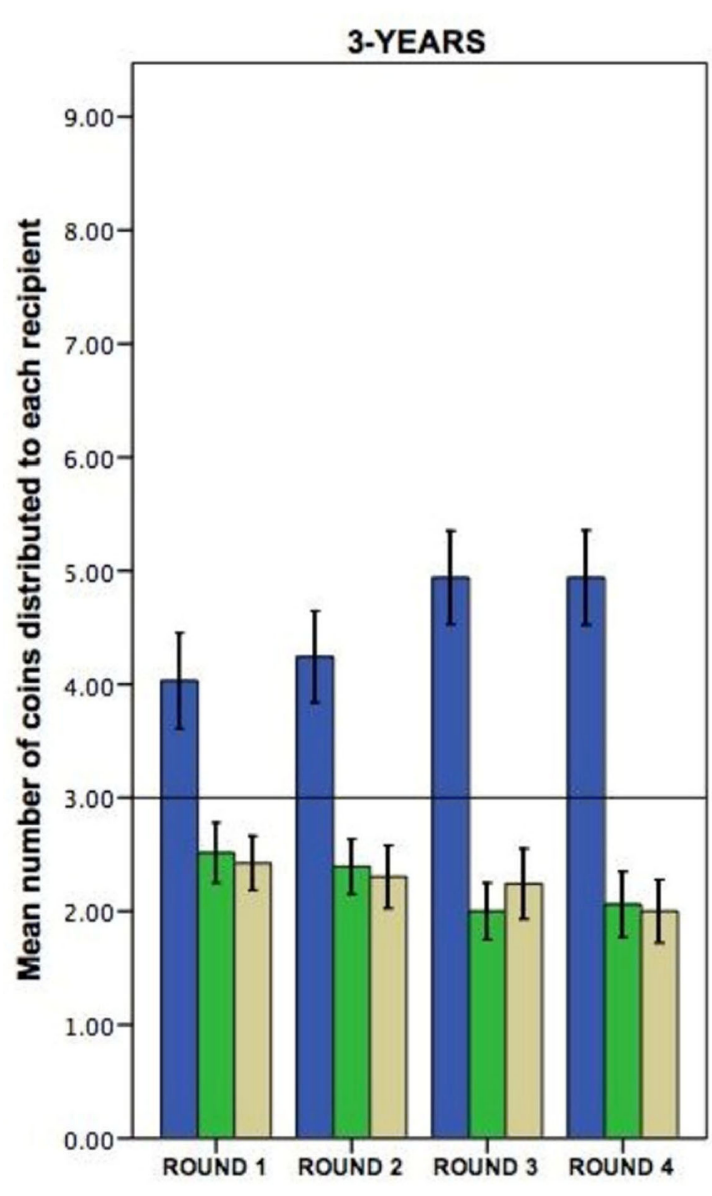

FIGURE 3 | Children's distributions in triadic sharing in Study 2.

Vertical axis represents mean number of coins (maximum nine)

distributed by 3-year-olds (left panel) and 5-year-olds (right panel) to each
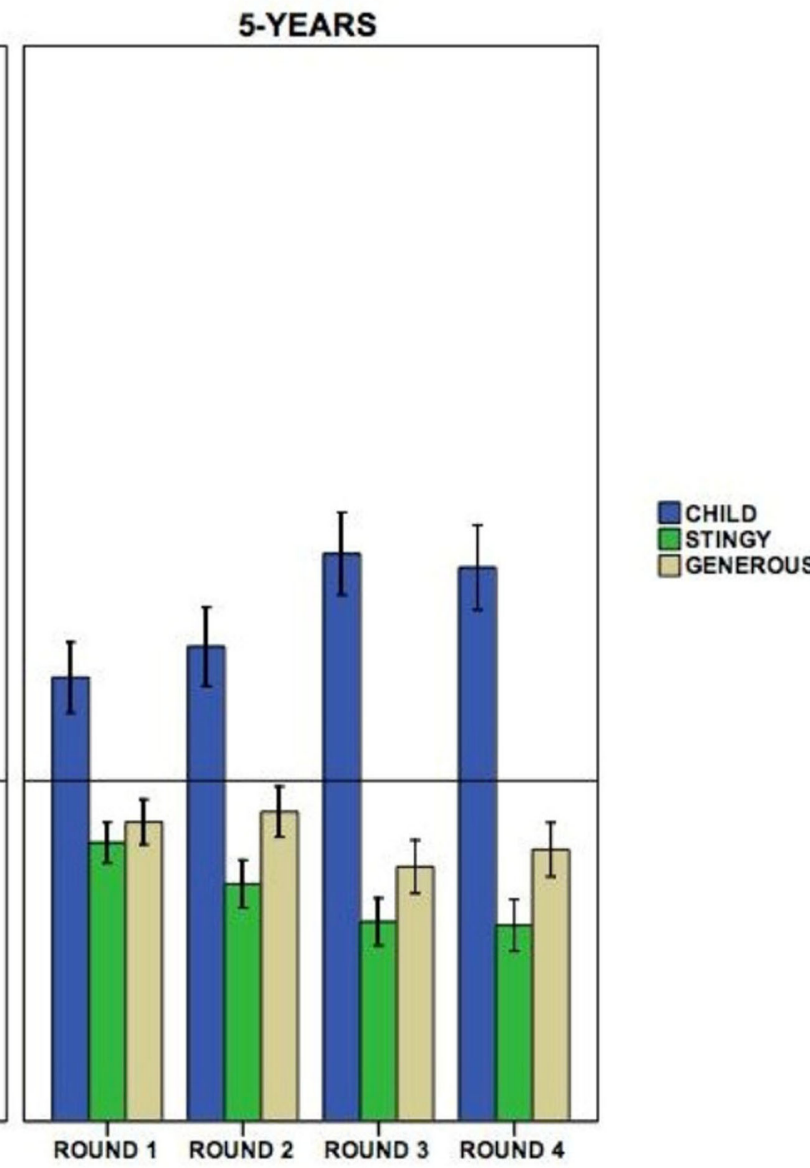

protagonist (child, stingy, and generous) as a function of round (1-4). The horizontal line represents absolute equity (three coins). Bars represent \pm 1 SE.
In the follow-up costly punishment task, the inclination to engage in costly punishment did not differ between age groups: $76 \%$ of 3 -year-olds $(N=25)$ and $91 \%$ of 5 -year-olds $(N=30)$ opted to punish at least once. Because the puppets differed with regard to their stingy or generous acts, we hypothesized that fairminded children should be less inclined to equally punish both puppets. A two-tailed Fisher's exact test compared the percentage of children in each age group who punished in successive attempts both puppets. Sixty-four-percent of 3-year-olds did so, compared to only $17 \%$ of 5 -year-olds, $p<0.01$.

To further investigate the selective orientation of children's costly punishment as a speculative expression of strong reciprocity, we used a linear regression model with the child's age (in months) to predict (out of all coins sacrificed) the percentage of coins given up to punish the stingy puppet. We observed a significant, moderate to strong association $\left(R \_1,55^{2}=0.320, p<0.01\right)$ between age and punishment orientation. These data suggest that as a function of age, when children sacrifice coins, they are increasingly selective in orienting their punishment toward the stingy puppet. Note that by 60 months, fewer children punish both puppets and no children punish the generous puppet only (Figure 5, left panel).
Collectively, the results of the costly punishment task indicate that unlike 3-year-olds, 5-year-olds selectively orient punishment toward the stingy character. We interpret this as first signs of strong reciprocity.

\section{ALTERNATIVE HYPOTHESES AND FURTHER ANALYSES}

Regarding the triadic sharing game, the differing allocations by the child to the two protagonists could rest on straight imitation (e.g., copying identically the actions of the stingy or generous puppet). Children might also focus on the overall unequal accumulation of coins in the banks that were transparent, hence public. Because the generous puppet always receives the least amount of coins prior to the child's turn, the number of coins in this bank is typically less than that of the other two protagonists. It is therefore possible that children gave more coins to the generous puppet in order to rectify this perceptual inequity (inequity aversion hypothesis), rather than as a means of expressing approval or spite for the puppet's generous versus stingy actions (ethical stance hypothesis).

To test these two alternative accounts, we identified 13 potential ways for children to distribute coins during the triadic share. To probe the alternative imitation account, we analyzed the extent 
to which children imitated the stingy and generous puppet in a strict "tit-for-tat" manner (i.e., giving to each of puppet the exact number of coins that the child's received from each) during any of four rounds of triadic sharing. We found that such emulation of either puppet was evident only for a small minority of children at both ages ( $22 \%$ of 3 -year-olds and only $5 \%$ of 5 -year-olds). There was no significant age difference in this trend based on chi-square calculations $(p=0.143)$. We therefore conclude that straight imitation alternative cannot account for our results.

To probe the inequity aversion hypothesis we reasoned that if children are responding based on a perceived perceptual inequity, then the most common sharing strategies should be those in which children favor the generous puppet to level the number of coins in the three banks (generous-oriented sharing). Children were only coded as engaging in generous-oriented sharing if they gave systematically more to the generous compared to either the self or the stingy puppet. Self-maximizing or stingy oriented strategies were therefore incompatible with such sharing (mutually exclusive). Analysis reveals that generous-oriented strategies were uncommon, with $13 \% 3$-year-olds and only $8 \%$ of 5 -year-olds sharing this way ${ }^{3}$. The inequity aversion hypothesis does not seem to hold.

Finally, in relation to costly punishment, we analyzed whether children engaged in costly sacrifice not to punish proper, but rather to re-establish equal distribution among the protagonists (inequity aversion hypothesis). We calculated an index of inequity by computing for each participant the proportion of coins in each bank at the end of the four round distributions (total accumulated coins in stingy's bank/(total accumulation of coins in child's bank + total accumulated coins in generous's bank). As a result, and considering mathematical lower and upper limits, the index of inequity could vary between a minimum value of 0.68 and a maximum value of 2.86, with greater values indicating greater levels of inequity benefiting the stingy puppet (maximum possible accumulation for the stingy puppet which was 80 coins). We reasoned that the inequity aversion hypothesis would be supported if the index of inequity (as it emerges at the end of the four rounds of distribution and depending only on the child's distribution) would (1) positively correlate with each child's inclination to engage in costly punishment and (2) would positively correlate with each child's inclination to punish the doll that typically ended up with the most coins (i.e., the stingy puppet).

Non-parametric correlation test between our index of inequity and whether or not the child opted toward costly punishment yielded non-significant results for both age groups, respectively $\mathrm{rs}_{31}=-0.15$ for 3 -year-olds and $\mathrm{rs}_{31}=-0.25$ for 5 -year-olds. Parametric correlation tests between index of inequity and the proportion of all coins sacrificed to punish the stingy puppet, also yielded non-significant results for both age groups, respectively $r_{31}=0.018$ for 3 -year-olds and $r_{31}=0.287$ for 5 -year-olds. From these results, we conclude that the inequity aversion hypothesis cannot readily account for our costly punishment results.

\footnotetext{
${ }^{3}$ Percentages for the sharing patterns in Study 1 were comparable and did not differ significantly from those reported here for Study 2.
}

To assert that children are indeed more or less sensitive to the stingy or generous character of the puppets, we performed three control experiments that are presented next. Specifically, we repeated the triadic share and costly punishment task with three new cohorts of children sharing in turn with either (a) two identical generous puppets (Study 3), (b) two identical stingy puppets (Study 4), or (c) puppets that were non-agentive in the splitting of coins (Study 5).

\section{STUDY 3 (GENEROUS CONTROL TRIADIC SHARE AND COSTLY PUNISHMENT)}

To determine whether children are sensitive to the generous character of the puppet, we ran a first control sampling 24 children (12 three-year-olds and 12 five-year-olds, see Table 1) who played the Triadic Sharing game and Costly Punishment task described previously, but with identically generous puppets.

\section{HYPOTHESES}

Regarding the four rounds of three-way sharing with the two generous puppets, we anticipated that 5-year-olds would show evidence of reciprocity by decreasing their degree of self-maximizing, compared to 3-year-olds who should persist in self-maximizing regardless of both puppets' generous characters. In relation to the costly punishment task, because both puppets acted with marked generosity (hence negating any need to adopt a principled "ethical stance"), we expected a lesser frequency of costly punishment compared to Study 2. Furthermore, we predicted that if punishment did occur, it would not be oriented systematically toward either one of the generous puppets.

\section{RESULTS}

Data were analyzed using the same 4 (round) $\times 3$ (recipient) $\times 2$ (age) $\times 2$ (gender) mixed-design ANOVA described previously. Because children distributed coins between themselves and identical generous protagonists, the potential recipients for this study included the child, the left-side puppet, and the right-side puppet. Gender and children's performance on the three-way pre-test (included as a covariate) were both non-significant, $F_{1,19}=0.479$ and 0.373 , respectively.

Analysis yielded a significant interaction of recipient and age, $F_{2,18}=4.83, p=0.021, \eta^{2}=0.349$. Contrasts reveal that as recipients and with regard to self-maximizing tendencies, 3-yearolds gave more coins to themselves $(M \pm \mathrm{SD}=4.17 \pm 2.02)$ than did 5-year-olds $(M \pm \mathrm{SD}=2.27 \pm 1.27), F_{1,19}=5.24, p=0.034$, $\eta^{2}=0.216$. Three-year-olds also tended to give more coins to themselves than to either the left $(M \pm S D=2.18 \pm 1.97)$ or right $(M \pm \mathrm{SD}=2.76 \pm 1.67)$ puppets, both $p<0.05$ based on Bonferroni-adjusted pairwise comparisons. Amongst 5year-olds there was a non-significant trend for children to give more coins to the left $(M \pm S D=3.40 \pm 1.79)$ and to the right puppet $(M \pm \mathrm{SD}=3.34 \pm 1.56)$ than to themselves $(M \pm \mathrm{SD}=2.27 \pm 1.28)$, suggesting that these children were sharing with almost absolute equity. Neither 3- nor 5-yearolds exhibited signs of a side bias by preferentially giving significantly more coins to the right versus the leftside puppet (see Figure 4, left and right panels, respectively). 


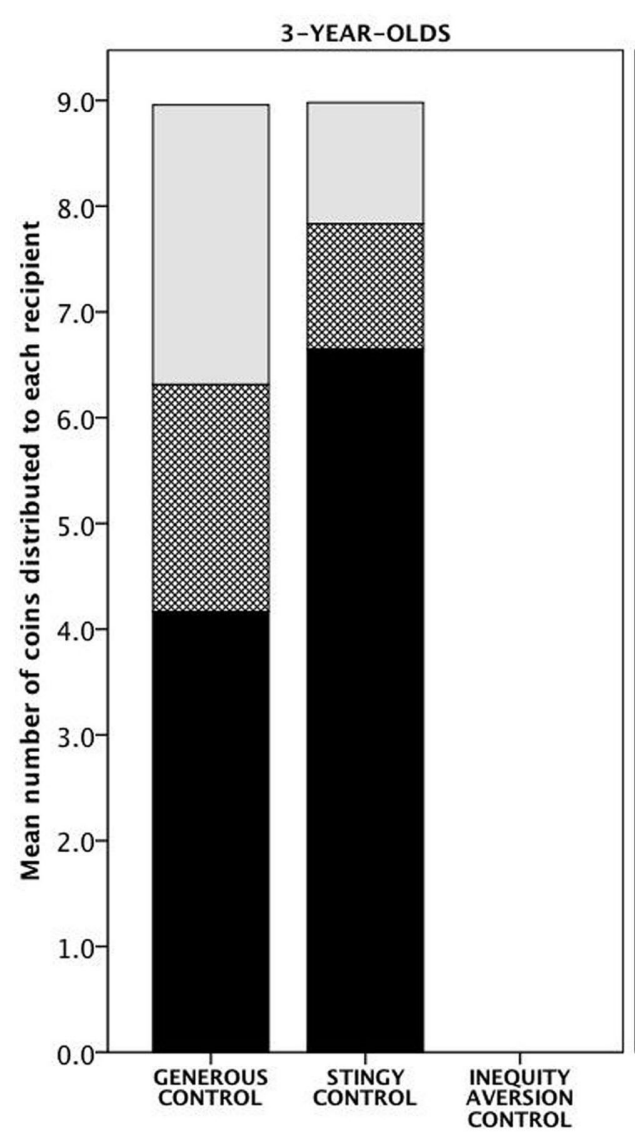

FIGURE 4 | Children's distributions in triadic sharing in Study 3 (Generous Control), Study 4 (Stingy Control) and Study 5 (Non-Agentive Control). Children split coins between themselves and two puppets that were either identically generous (Study 3), or stingy (Study 4), or were passive recipients of the game (Study 5). Vertical axis

\section{5-YEAR-OLDS}

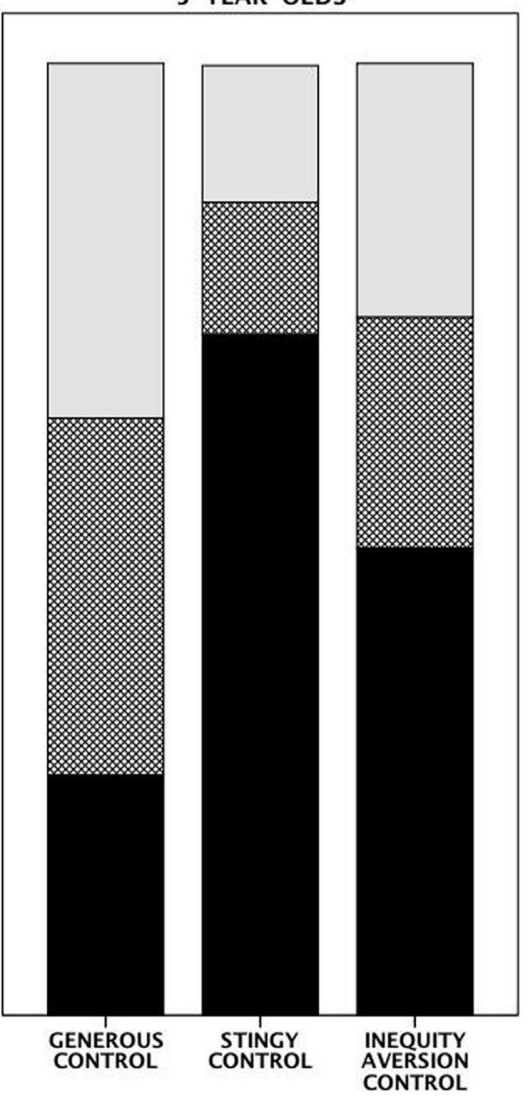

口GENEROUS (or RIGHT-SIDE) QSTINGY (or LEFT-SIDE) CHILD
Note that despite these developmental differences, both 3- and 5 -year-olds in this Study were on average less self-maximizing than their counterparts in Study 2. We computed a "self-maximizing" score by averaging the number of coins given to the child across the four sharing rounds and used this score as the dependent measure in an independent samples $t$-test to compare self-maximizing across the two studies. Overall, children in Study 3 (Generous Control) gave significantly fewer coins $(M \pm \mathrm{SD}=3.21 \pm 1.92)$ to themselves than did children in Study 2 (Stingy-Generous Triadic Share, $M \pm \mathrm{SD}=4.53 \pm 1.86$ ), $t_{88}=2.95, p<0.01$ (one-tail).

These findings suggest that even when sharing with identical generous protagonists, 3-year-old children persist in their maximization of personal gains; their sharing in this control condition was akin to the self-maximizing observed in 3-year-olds in both Studies 1 and 2. In contrast, and confirming our hypothesis, 5year-olds showed clear signs of reciprocity with the puppets by sharing more generously and self-maximizing significantly less compared to Study 2.

In the absence of any overtly unfair sharing on the part of the two puppets, we did not expect children at either age to represents mean number of coins (maximum 9) distributed by 3-year-olds (left panel) and 5-year-olds (right panel) to each recipient collapsed across round. Note that to control for the possible effect of inequity aversion in older children, only 5-year-olds were tested in Study 5. engage in costly punishment. Owing to the identical character of the puppets, if costly punishment occurred, we expected the orientation of such punishment to be at chance. A series of Fisher's exact tests supported these predictions. Approximately half of $3-(58 \%, N=7)$ and 5 -year-olds $(42 \%, N=5)$ engaged in costly punishment; overall, this cohort punished significantly less frequently than did children in Study 2, Fisher's exact test: $p<0.01$ (one-tail). Furthermore, when children punished they did so without clearly aligning punishment toward one puppet. Note that $71 \%$ of 3 -year-olds and $60 \%$ of 5 -year-olds punished both puppets. Linear regression demonstrated that the association between children's age (in months) and the percentage of coins (out of all coins sacrificed) given to punish the left puppet was non-significant, $R \_1,10^{2}=0.02$ (Figure 5, center panel). That 5-year-olds show no signs of selective costly punishment is in sharp contrast to the findings reported in Study 2. We speculate that when they punish in this control study, children of both age groups act instead of punishing proper to prolong the playful exchange with the Experimenter (ludic inertia, see General Discussion). 
In all, Study 3 confirms that between 3 and 5 years, children become more sensitive to the generous character of sharing protagonists, aligning with their generous tendencies in acts of reciprocity. Arguably, in the absence of any overt violation of fairness norms on the part of the two generous puppets, children were not motivated to adopt an ethical stance, opting to engage in selective costly punishment only at chance levels. For further control, in Study 4, we repeated the experiment with two stingy puppets.

\section{STUDY 4 (STINGY CONTROL WITH TRIADIC SHARE AND COSTLY PUNISHMENT)}

To assert that children are indeed sensitive to the stingy character of the puppet, in a second control condition we sampled 24 children (12 three-year-olds and 12 five-year-olds, see Table 1) who played the Triadic Sharing Game and Costly Punishment task described previously with two identically stingy puppets.

\section{HYPOTHESES}

Regarding the four rounds of three-way sharing with identical stingy puppets, we expected that if 5-year-olds are engaging in reciprocity, they should increase their tendency to self-maximize, more closely resembling 3-year-olds and giving themselves more coins compared to Study 2. For the costly punishment task, we anticipated at both ages no evidence of selective punishment toward either one of the two puppets owing to the identical nature of their characters (i.e., stingy character in both).

\section{RESULTS}

Because children distributed coins between themselves and identical stingy protagonists, potential recipients of sharing included the child, the left puppet, and the right puppet. Triadic share data were analyzed using a 4 (round) $\times 3$ (recipient) $\times 2$ (age) $\times 2$ (gender) mixed-design ANOVA. Gender and performance on the three-way pre-test (included as a covariate) were non-significant, $F_{1,18}=2.82$ and 0.565 , respectively.

A main effect of recipient $\left(F_{2,17}=55.8, p<0.01, \eta^{2}=0.112\right)$ demonstrates that 3 - and 5 -year-olds were similar in how they distributed coins amongst the three protagonists (Figure 4, left and right panels, respectively). Children at both ages maximized their gains, giving themselves on average 6.5 (roughly $72 \%$ ) of the nine coins. Mean differences indicate that children gave significantly more coins to themselves than to either the left $(\mathrm{MD} \pm \mathrm{SE}=5.45 \pm 0.402)$ or right $(\mathrm{MD} \pm \mathrm{SE}=5.42 \pm 0.452)$ puppet, both $p<0.01$ based on Bonferroni-adjusted pairwise comparisons. Neither 3- nor 5-year-olds exhibited signs of a side bias by preferentially giving significantly more coins to the right versus the left-side puppet (see Figure 4, left and right panels, respectively).

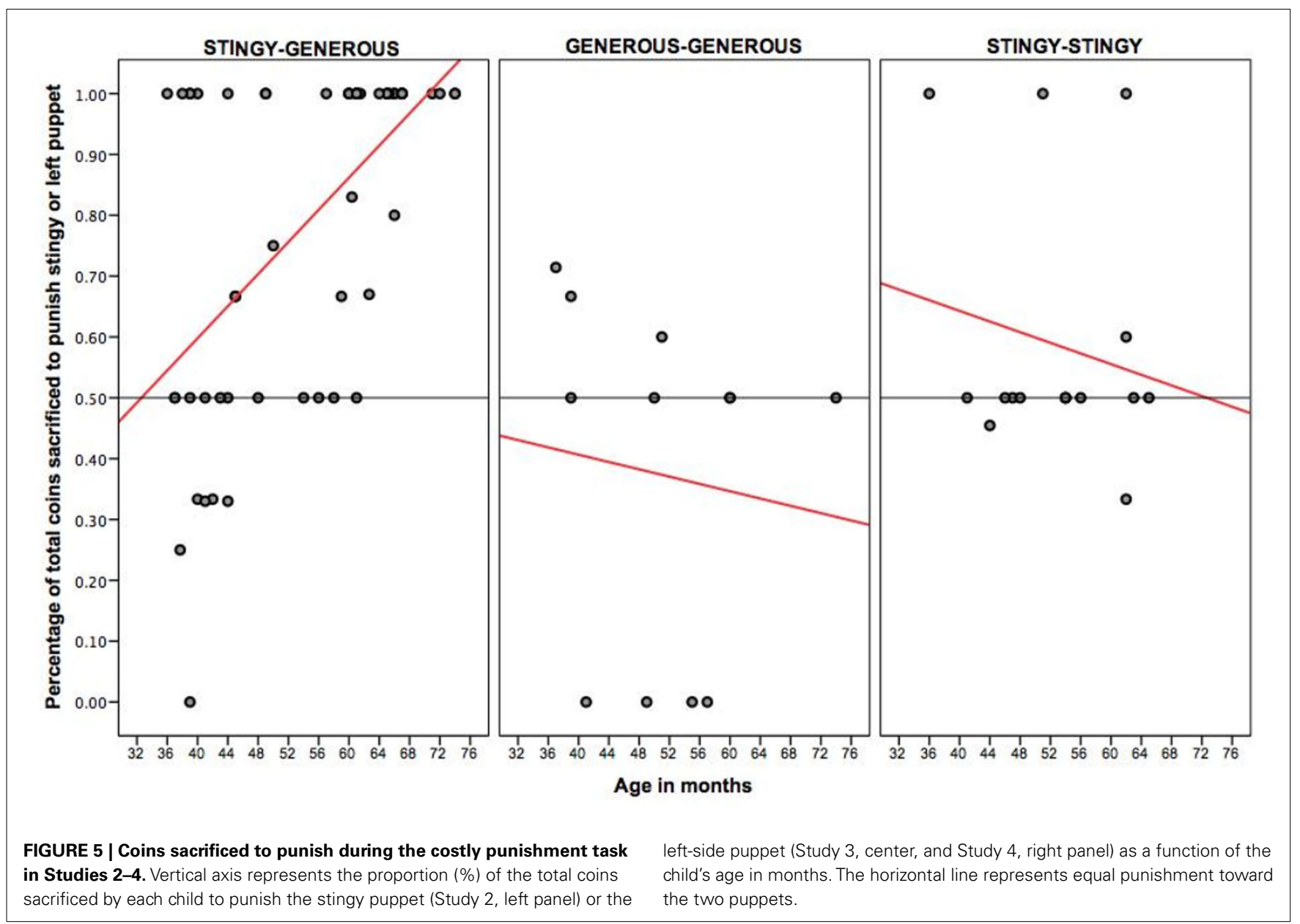


Children's degree of self-maximizing in this condition was elevated even above levels noted in Study 2. To compare across studies, we first computed a "self-maximization" score by averaging together the number of coins distributed to the child across the four sharing rounds. This value was entered as the dependent measure in an independent samples $t$-test. Regardless of age, when in the presence of two stingy protagonists children give significantly more coins to themselves $(M \pm \mathrm{SD}=6.52 \pm 1.33)$ than in situations in which they distribute resources with a stingy and a generous puppet, as in Study $2(M \pm \mathrm{SD}=4.53 \pm 1.86)$, $t_{88}=4.83, p<0.01$ (one-tail).

Consistent with the generous control condition of Study 3, both 3- and 5-year-olds were at chance regarding the inclination to engage in costly punishment. Overall, children in this cohort were significantly less likely to punish than were children in Study 2, Fisher's exact test: $p<0.01$ (one-tail). Furthermore, children who punished were non-selective in their orientation of punishment: $67 \%$ of 3 -year-olds and $83 \%$ of 5 -year-olds punished both puppets equally often. A linear regression model testing the relationship between children's age (in months) and the percentage (out of total sacrificed coins) given to punish the left puppet yielded a non-significant association, $R \_1,15^{2}=0.05$ (see Figure 5, right panel).

In all, the results confirm our prediction that when sharing with two stingy characters, both 3- and 5-year-old children demonstrate strong self-maximizing tendencies. As in Study 3 (generous control) but in contrast to Study 2 (stingy and generous partners), 5 -year-olds show no sign of selective costly punishment when agreeing to sacrifice one of their coins at the end of the sharing game.

To further control that 5-year-olds do construe and factor the stingy and generous characters of the puppets as sharing agents, we performed a final control with non-agentive (passive) puppets. The rationale was that if 5-year-olds were only considering the outcome of the sharing (i.e., unequal coin distribution independent of the agentive character of the puppet), we should replicate the findings of Study 2. If not, the strong reciprocity (ethical stance) hypothesis would find further support to account for the behavior of 5-year-olds.

\section{STUDY 5 (NON-AGENTIVE CONTROL WITH TRIADIC SHARE AND COSTLY PUNISHMENT)}

In a final control we tested the possibility that sharing outcome (e.g., the number of coins in the banks at the end of the Triadic Sharing game) rather than the character and agency of the puppets could account for signs of strong reciprocity. In Study 5 , children playing the same Triadic Sharing game described previously were presented with a pre-established distribution, never seeing the puppets actually sharing either stingily or generously but resting inanimate in front of the child. Prior to the child's turn to split the coins, the Experimenter displayed three groups of coins on a tray, one for each of the three protagonists. The coins were presented in the same ratios as in Studies land 2. Thus, in one turn the "stingy" puppet received four coins while the child and "generous" puppet received only one each, and in another turn the "generous" puppet received one coin while the child and "stingy" puppet each received four coins. As in the previous studies, we counterbalanced the location of the two puppets relative to the child as well as the order in which the stingy and generous distributions were presented. When it was the child's turn, the child actively distributed as in the other studies. At the end of the Triadic Sharing game children participated in the same Costly Punishment task described previously. Because in Study 2 selective costly punishment oriented toward the stingy protagonist was only observed in older children, we limited our sample in this new control condition to only 5-year-olds (see Table $\mathbf{1}$ ).

\section{HYPOTHESIS}

Following the rationale of this control (see above) and based on the strong reciprocity hypothesis demonstrated in 5-year-olds (Study 2 ), we expected significantly less signs of oriented costly punishment in this non-agentive, passive puppet condition (no clear agentive character of the puppets).

\section{RESULTS}

Once again, triadic share data were analyzed using a 4 (round) $\times 3$ (recipient) mixed-design ANOVA. On the basis of our earlier findings, gender and the pre-test were omitted as factors in analysis. Results yielded a main effect of recipient, $F_{2,14}=5.566, p=0.017$, $\eta^{2}=0.263$. On average children gave significantly more coins to themselves $(M=4.521, \mathrm{SD}=0.484)$ than to either the stingy $(M=2.118, \mathrm{SD}=0.313)$ or generous $(M=2.361, \mathrm{SD}=0.264)$, both $p<0.05$ based on Bonferroni-adjusted pairwise comparisons. Unlike their counterparts in Study 2, children in this control condition were less self-maximizing and did not discriminate between the two non-agentive puppets (Figure 4, right panel).

With regards to the costly punishment task, a binomial test revealed that significantly more children $(N=14$, or $78 \%)$ opted to punish than not, $p=0.015$ (one-tail). This value did not differ significantly from the $91 \%$ of 5 -year-olds who opted to punish in Study 2 based on a Fisher's exact test.

However, differences emerged with regard to the selectivity of punishment. In this control condition, 7 of 14 children (50\%) punished both puppets. In contrast, children in Study 2 were more selective: $17 \%$ of children punished both puppets, whereas the majority $(83 \%)$ were significantly above chance levels in their punishment of only one puppet, $p<0.05$. In a more direct Fisher's exact test of these two studies, our results show a significant trend by which children in Study 2 were more likely to orient punishment toward only one puppet than were children in the current control, $p<0.05$. However, when exclusive punishment of one puppet did occur, results show that, as in Study 2 , it was oriented toward the stingy protagonist. Of the children who sacrificed coins to punish only one puppet, $100 \%$ of children in Study 2 and $86 \%$ of children in the present control oriented this punishment toward the stingy puppet. The percentage of coins sacrificed to punish the stingy puppet was also similar between Study $2(M \pm \mathrm{SD}=0.827 \pm 0.285)$ and the present control $(M \pm \mathrm{SD}=0.680 \pm 0.337), t_{42}=1.334, p=0.193$ (two-tailed). In short, significantly more children punished both puppets in the control compared to Study 2. However, when children opted to punish only one puppet, in both studies the majority of children punished the stingy protagonist. 
As in Study 2, to determine whether punishment oriented toward the stingy protagonist could be predicted by the total accumulated coins in each of the three banks, we correlated our index of inequity aversion with the percentage of coins sacrificed to punish the stingy puppet (see Study 2 for formulation). Consistent with the results of Study 2, this relationship was non-significant, $r(14)=0.023$, suggesting that the differing amounts of coins in each recipient's bank at the end of the fourth round did not predict selective punishment toward the stingy character.

These results suggest that in the absence of any agency or explicit reference to the character of the two puppets, children were significantly less inclined to orient their punishment solely to the stingy or the generous protagonist, in contrast to what we found in Study 2. Furthermore, in both studies, when children opted to punish the Stingy puppet at least once, this tendency was not predicted by the relative number of coins in each recipient's bank at the end of the game. We therefore conclude that the inequity aversion hypothesis cannot account for our results.

\section{STUDY 6 (CROSS-CULTURAL COMPARISON OF US AND SAMOAN 5-YEAR-OLDS)}

Egalitarianism and responses to unfair or inequitable acts might be deeply rooted in human evolutionary history (Fehr et al., 2008; Olson and Spelke, 2008). Such claims call for further cross-cultural comparisons that would confirm the universality of strong reciprocity as they emerge in human ontogeny. We had the rare opportunity to test a sample of 5- to 6-year-olds living in a small rural and traditional Polynesian village of Samoa in the South Pacific (see description below). Here we present findings for both the triadic share and costly punishment replicating the procedure outlined in Study 2. We compared Samoan children with age and gendermatched children from our sample of American 5-year-olds in Study 2. The rationale was to probe the role of highly contrasted cultural contexts on children's early expression of strong reciprocity and to assess the extent to which such development might be universal.

\section{SAMOAN CHILDREN'S CULTURAL CONTEXT}

Children were recruited from the village of Faga (population approximately 500) on the East coast of the island of Savai' $i$ (population approximately 50,000) of independent Samoa (population approximately 180,000 , with a GDP nominal per capita of $\$ 2,608$ USD, as opposed to $\$ 46,900$ in the United States). Samoa is at the heart of Polynesia and still maintains strict ancestral Polynesian traditions despite successive colonial dominations by the British, Germans, and New-Zealanders, notwithstanding a widespread conversion to Christian churches of almost all denominations starting the middle of the nineteenth Century. Gaining its political independence in 1962, and despite years of Western influence and governance, Samoa has managed to uphold strong traditions of collective living organized around a highly hierarchical chief ("Mataii") system that is typical of Polynesian culture. In Samoa, this system is maintained in arguably its purest form within the Polynesian context of the South Pacific (Shore, 1982; O'Meara, 1990). Samoan culture and societal organization is a good example of what is sometimes characterized as "vertical" as opposed to "horizontal" or "egalitarian" collectivism (Triandis and Gelfand, 1998).
Children were tested in the village of Faga, which engages in horticulture, fishing, and marine foraging for survival and as a means of small cash revenue, aside from remittance money sent from family members living and working abroad, primarily in New Zealand. Before children attend school (at around age three to five), they typically spend the day with their immediate and extended family, playing outdoors with other young children, or observing alongside adults as they perform their daily duties. There is minimal adult supervision after the child begins to walk; however children tend to be supervised collectively by the adults and older children in the village. Aside from collective games around balls and very few other shared objects, these children develop in a culture that does not emphasize individual possession. Children toys are rare in the Samoan context that emphasizes communal properties and living space, rather than individual possession and private quarters.

\section{METHODOLOGICAL CONSIDERATIONS AND PARTICIPANTS}

We tried to replicate with Samoan 5- to 6-year-old children the multi-round, triadic sharing game with stingy and generous puppets as well as the costly punishment task described in Study 2. In Samoa we used materials that were more familiar to the participants such that children split buttons between two sock puppets, props that were meant to be culturally less eccentric than the poker coins and the high-quality plush puppets used with the US children. Consistent with Studies 1-4, children were informed that, like the coins, buttons were valuable placeholders to be redeemed at a makeshift toy store at the game's conclusion. Figure 6 depicts a child tested in Samoa.

We tested a 14 children (seven male and seven female, see Table 1). All children were tested by a trained adult native from the village who was fluent in Samoan language. Back translations of the Samoan Experimenter's instructions to the child were performed to ensure consistency and comparability between Samoan and English.

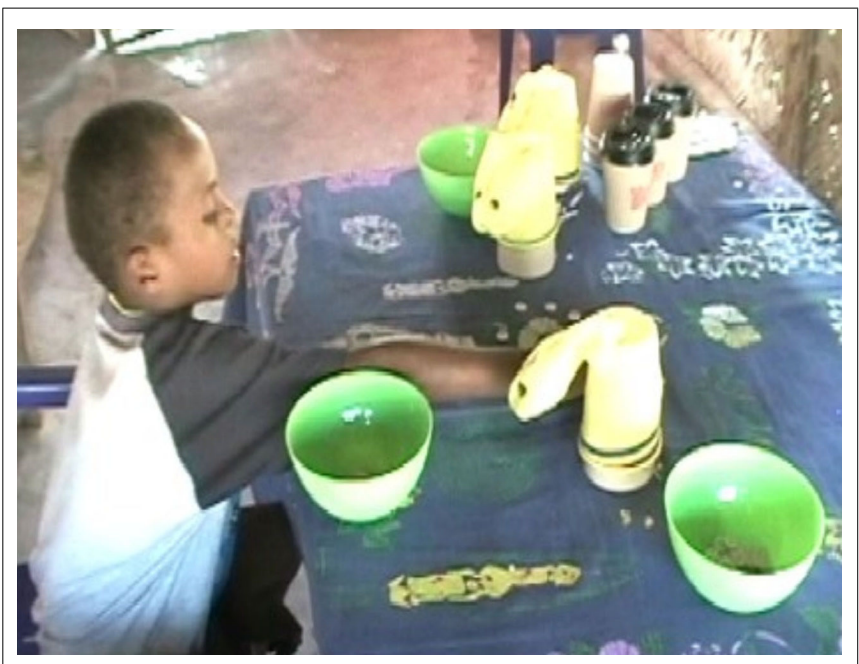

FIGURE 6 | Experimental situation in Samoa. The plush puppets and poker chips used for the American samples were replaced with more culturally familiar items (sock puppets and buttons). 


\section{RESULTS}

For the triadic sharing game, the number of buttons (out of nine) distributed to each player was analyzed using a 4 (round) $\times 3$ (recipient) repeated measures ANOVA. Gender and performance on the three-way pre-test were not included as factors because there was very little variation in sharing between males and females, and all but 1 child in the Samoan sample shared coins equitably at pre-test. Similarly, because our sample was limited to 5-year-olds, age was not factored into the analysis.

We observed a main effect of recipient, $F_{2,12}=4.86$, $p=0.029, \eta^{2}=0.442$. Pairwise comparisons indicate that in general, unlike American children, Samoan children gave fewer coins to themselves $(M \pm \mathrm{SD}=2.29 \pm 0.282)$ than to either the stingy puppet $(M \pm \mathrm{SE}=3.46 \pm 0.265)$ or the generous puppet $(M \pm \mathrm{SD}=3.21 \pm 0.175)$, both $p<0.05$ (Bonferroniadjusted). Also in contrast to the American 5-year-olds of Study 2, our Samoan sample did not discriminate between the two puppets, giving roughly the same number of coins to both generous and stingy protagonists, $\mathrm{MD} \pm \mathrm{SE}=-0.167 \pm 0.241$.
With regard to costly punishment, Samoan 5-year-olds opted to punish at chance levels: Eight children (57\%) engaged in costly sacrifice whereas six children (43\%) did not, both $p>0.05$ based on binomial tests. When they punished, children were also at chance regarding who should be punished, with approximately half of the children punishing stingy alone, and half punishing both characters.

\section{DIRECT COMPARISON WITH THE US SAMPLE}

We compared our sample of Samoan 5-year-olds (Figure 7, left panel) to age and gender-matched, randomly chosen 5-year-olds from our sample of American children in Study 2 (seven males and seven females, see Table $\mathbf{1}$ ).

Regarding the triadic share (see Figure 7), we computed a "self-maximizing" score for our matched sample of Samoan and American 5-year-olds by averaging the number of coins distributed to the self across the four sharing rounds. This score was then used in an independent samples $t$-test. Results show that American children gave themselves significantly more coins $(M \pm \mathrm{SD}=4.70 \pm 1.55)$ than did Samoan children $(M \pm \mathrm{SD}=2.29 \pm 0.976), t_{26}=4.55, p<0.01$ (one-tailed).

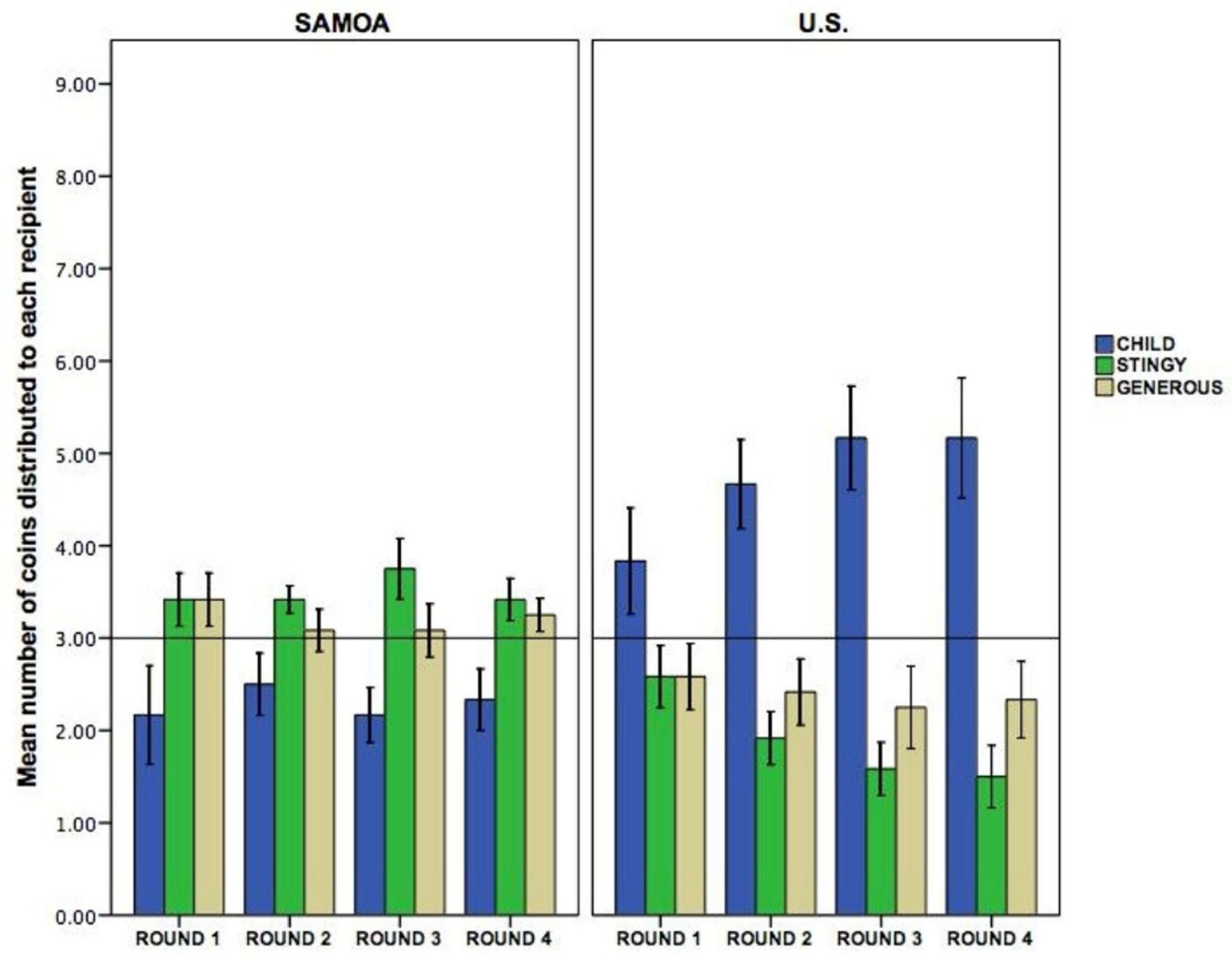

FIGURE 7 | Samoan and American 5-year-olds' distributions in triadic sharing in Study 6. Vertical axis represents mean number of coins (maximum 9) distributed by Samoan children (left panel) versus a randomly selected sample of age and gender-matched children from Study 2 (right panel) to each protagonist (child, left puppet, right puppet) as a function of round (1-4). The horizontal line represents absolute equity (three coins). Bars represent $\pm 1 \mathrm{SE}$. 
Samoan 5-year-olds also differed in their treatment of the two puppets. We analyzed the number of coins distributed to protagonists in a 4 (round) $\times 2$ (recipient: stingy or generous) $\times 2$ (culture) mixed-design ANOVA, finding a significant interaction of culture and recipient, $F_{1,26}=8.31, p=0.027$, $\eta^{2}=0.225$. Across the four sharing rounds, American children preferentially shared with the generous over the stingy puppet, $\mathrm{MD} \pm \mathrm{SE}=0.492 \pm 0.176, p<0.01$ (based on Bonferroniadjusted pairwise comparisons). In contrast, although Samoan children gave slightly more coins to the stingy puppet, the mean difference in the number of coins distributed to the puppets was non-significant, $\mathrm{MD} \pm \mathrm{SE}=-0.167 \pm 0.241$. Samoan children did not preferentially share with either puppet.

With regard to costly punishment, a Fisher's exact test reveals a non-significant trend of more American children $(86 \%, N=12)$ opting to punish compared to $57 \%(N=8)$ of Samoan children. When children agreed to give up coins to punish, the percentage sacrificed to punish the stingy puppet did not differ significantly across cultures, $M \pm S D=0.817 \pm 0.333$ for the US and $M \pm \mathrm{SD}=0.576 \pm 0.348$ for Samoa, based on an independent $t$-test: $t_{18}=1.48, p>0.05$. However, when examining each culture independently, only US children were above chance levels ( $p=0.005)$ in choosing to punish, based on binomial tests. These finding suggests that although punishment may be less common in Samoa, when punishment does occur, like in the US, it tends to be oriented more toward the stingy puppet. However, note that because of the small sample size, there might be insufficient power to detect a statistically significant difference between the two cultures. Alternatively, Samoan children may be less inclined to punish, but also less inclined to engage in strong reciprocity oriented toward a protagonist who has violated an expected norm of fairness. It is possible that norms of fairness might differ between Samoan and US children. More research is necessary to further assess the relative universality of strong reciprocity, with a focus on the relationship between cultural context and the inclination to selectively punish. This said, our preliminary investigation confirms that there might be important, yet subtle cross-cultural differences.

\section{GENERAL DISCUSSION}

Collectively, our findings provide first evidence that what we construe as strong reciprocity emerges by 5 years in human ontogeny, at least in middle class US children. Although 3-year-olds modulate their tendency toward self-maximizing depending on the relative generosity or stinginess of sharing partners, this early sensitivity is not yet principled. In 3-year-olds, there are no apparent signs of selective costly punishment. Our research suggests that signs of strong reciprocity emerge from 5 years of age.

Furthermore, our analysis shows that a simple aversion to inequity cannot account for what we observe. If some sensitivity to inequity is necessary for the expression strong reciprocity, is not sufficient. In the expression of strong reciprocity as construed here, individuals not only detect but also tend to act principally upon it by either punishing or rewarding, even if doing so comes at a personal cost. The developing propensity to act, even at a personal cost, captures first signs of an ethical, morally principled stance emerging by 5 years of age and not earlier.
The results of the triadic sharing game in Studies 1 and 2 suggest that although children in both age groups express self-maximizing tendencies, only 5-year-olds demonstrate evidence of reciprocity by discriminating between the generous and stingy puppets, opting to give more coins to the generous puppet and reducing payoffs to the stingy puppet over the four rounds of sharing. In the two follow-up control conditions, 5-year-olds showed further signs of reciprocity by decreasing their self-maximizing behavior when sharing with identical generous puppets (Study 3 ) and increasing this self-maximization when interacting with identical stingy puppets (Study 4). When sharing with non-agentive characters (Study 5), 5-year-olds self-maximized their payoffs (though to a lesser extent than in Studies 1 and 2) and did not discriminate significantly between the "stingy" and "generous" protagonists who were passive actors and recipients in the game.

Study 2 provides further evidence that although children of both age groups engage in costly punishment, only 5 -year-olds show signs of strong reciprocity by orienting their costly sacrifice markedly more toward the stingy protagonist. Three-year-old children do not align punishment with either character, engaging in indiscriminate punishment that may be more about continuing the inertia of the game rather than conveying approval or disapproval for the puppets' actions. Selective costly punishment was only observed in conditions where children interacted with both an agentive stingy and an agentive generous protagonist. In control conditions where puppets were either identically generous (Study 3) or identically stingy (Study 4), both 3- and 5-year-olds were at chance with regard to the inclination to punish, and to orient punishment toward a particular protagonist. In the third control condition (Study 5), when the puppets were non-agentive and the distribution pre-established, 5-year-olds were significantly less inclined to punish only one of the two puppets, whether stingy or generous. In thus appears that by 5 years, children show significantly more signs of strong reciprocity. Our analyses also show that such signs of strong reciprocity cannot be merely reduced to an expression of inequity aversion. By 5 years, the costly behavior of the tested children cannot be predicted by the unequal accumulation of coins at the end of the triadic share. Instead, the inclination to engage in costly sacrifice seems to depend on the relative character of the child's sharing partners (i.e., stingy versus generous), particularly so when these partners behave as agents.

With regard to the relative universality and cross-cultural equivalence in the expression of strong reciprocity in ontogeny, a comparison of age-matched Samoan and American children (Study 6) suggests that the proclivity to engage in costly punishment is at 5 years of age more prevalent in the US compared to Samoa. Although further research is needed, our data suggest that culture could play a role in the expression of an ethical stance in child development. The collectivist, more grouporiented culture surrounding Samoan compared to US children, and particularly compared to the middle class US sample tested here, might impact their developing sense of fairness expressed in distributive justice. Samoan 5-year-old children did show a heightened tendency toward egalitarianism and lesser propensity toward self-maximizing in the context of our triadic sharing game. 
Questions remain as to what factors contribute to such development. Recent research suggests that concern for others in distributive justice games may be determined in part by parochialism and close relations (Fehr et al., 2008; Olson and Spelke, 2008). Favoritism toward perceived in-group members becomes a major predictors of fair distributions, particularly in children 5 years and older. The relationship between the development such distributive behavior and so-called moral emotions (e.g., spite, altruism) would need to be further investigated. Finally, evidence of thirdparty punishment (e.g., costly sacrifice to punish one who has wronged another) in 5-year-old children could strengthen they ethical stance hypothesis proposed here.

\section{CONCLUSION}

Beyond the speculation that humans might be born with a unique, species-specific "moral instinct," our own research points toward the importance of examining the role of culture in the expression of such fair-minded behavior and the child's ability to adopt an ethical stance. Preliminary cross-cultural differences reported here, although limited, point to various possibilities. For example, Samoan and US middle class cultures might be associated with different developmental trajectories regarding the emergence of

\section{REFERENCES}

Callaghan, T., Rochat, P., Lillard, A., Claux, M. L., Odden, H., Itakura, S., Tapanya, S., and Singh, S. (2005). Synchrony in the onset of mentalstate reasoning: evidence from five cultures. Psychol. Sci. 16, 378-384.

Damon, W. (1975). Early conceptions of positive justice as related to the development of logical operations. Child Dev. 46, 201-312.

Dupoux, E., and Jacob, P. (2007). Universal moral grammar: a critical appraisal. Trends Cogn. Sci. (Regul. Ed.) 11, 373-378.

Eisenberg, N., and Fabes, R. A. (1998). "Prosocial behavior and development," in Handbook of Child Psychology, ed. B. Damon (New York: Academic Press), 701-778.

Faigenbaum, G. (2005). Children's Economic Experience: Exchange, Reciprocity, and Value. Buenos Aires: Libros En Red.

Fehr, E., Bernhard, H., and Rockenbach, B. (2008). Egalitarianism in young children. Nature 454, 1079-1083.

Fehr, E., Fischbacher, U., and Gâchter, S. (2002). Strong reciprocity, human cooperation, and the enforcement of social norms. Hum. Nat. 13, $1-25$.

Haidt, J., and Joseph, C. (2004). Intuitive ethics: how innately prepared intuitions generate culturally variable virtues. Daedalus 133, 55-66.

Hamlin, J. K., Wynn, K., and Bloom, P. (2007). Social evaluation by preverbal infants. Nature 450, 557-560.

Hauser, M. (2006). Moral Minds. New York: Ecco.
Henrich, J., McElreath, R., Barr, A., Ensminger, J., Barrett, C., Bolyanatz, A., Cardenas, J. C., Gurven, M., Gwako, E., Henrich, N., Lesorogol, C., Marlowe, F., Tracer, D., and Ziker, J. (2006). Costly punishment across human societies. Science 312, 1767-1770.

Henrich, N., and Henrich, J. (2007). Why Humans Cooperate: A Cultural and Evolutionary Explanation. NY, Oxford: Oxford University Press.

Hook, J. (1978). The development of equity and logico-mathematical thinking. Child Dev. 49, 1035-1044.

Inhelder, B., and Piaget, J. (1964). The Early Growth of Logic in the Child. New York: Norton.

Kagan, J. (1988). Tempermental contributions to social behavior. Am. Psychol. 44, 668-674.

Keller, H., Yovsi, R., Borke, J., Kartner, J., Henning, J., and Zaira, P. (2004). Developmental consequences of early parenting experiences: self-recognition and selfregulation in three cultural communities. Child Dev. 75, 1745-1760.

Kohlberg, L. (1981). Essays on Moral Development. San Francisco: Harper \& Row.

Kuhlmeier, V. A., Wynn, K., and Bloom, P. (2003). Attribution of dispositional states by 12-month-olds. Psychol. Sci. 14, 402-408.

Lane, I. M., and Coon, R. C. (1972). Reward allocation in preschool children. Child Dev. 43, 1382-1389.

McCrink, K., Bloom, P., and Santos, L. R. (2009). Children's and adults

strong reciprocity. Alternatively, children of both cultures might express strong reciprocity at about the same age, but in different ways reflecting, for example, their relative inclination toward social conformity, their relative respect for rules and adult authority (Keller et al., 2004), as well as their greater sense of fairness and aversion toward inequality (Fehr et al., 2008; Rochat et al., 2009).

We conclude that strong reciprocity as the putative force behind human cooperation, as well as the human tendency to abide by and enforce social norms, might first emerge by 5 years of age and may depend on culture. From then on, children begin to situate themselves, their understandings, judgments, and actions toward others in a morally principled space (Taylor, 1989). This, we propose, represents a radical ontogenetic shift that is deserving of further empirical scrutiny.

\section{ACKNOWLEDGMENTS}

Our gratitude goes to Britt Berg and the students of the Emory University Infant and Child Lab for data collection, coding, and analysis. Our thanks also go to Tanya and James Broesch for helping in the conceptualization of the project in its early stages and for their assistance collecting data in Samoa.

judgments of equitable resource distributions. Dev. Sci. 13, 37-45.

Olson, K. R., and Spelke, E. S. (2008). Foundations of cooperation in young children. Cognition 108, 222-231.

O'Meara, T. (1990). Samoan Planters: Tradition and Economic Development in Polynesia. Fort Worth: Holt, Rinehart, \& Winston, Inc.

Piaget, J. (1932). The Moral Judgment of the Child. London: Routledge \& Kegan Paul.

Pinker, S. (2008). The Moral Instinct. New York Times Magazine, January 12.

Rochat, P., Dias, M. D. G., Guo, L. Broesch, T., Passos-Ferreira, C., Winning, A., and Berg, B. (2009). Fairness in distributive justice by 3 - and 5 -year-olds across 7 cultures. J. Cross Cult. Psychol. 40, 416-442.

Shore, B. (1982). Sala'ilua: A Samoan Mystery. New York: Columbia University Press.

Snarey, J. (1985). Cross-cultural universality of social-moral development: a critical review of Kohlbergian research. Psychol. Bull. 97, 202-232.

Taylor, C. (1989). The Sources of the Self: The Making of the Modern Identity. Cambridge, MA: Harvard University Press.

Tomassello, M. (2008). The origins of communication. Cambridge, MA: MIT Press.

Triandis, H. C., and Gelfand, M. J. (1998). Converging measurement of horizontal and vertical individual- ism and collectivism. J. Pers. Soc. Psychol. 74, 118-128.

Warneken, F., Hare, B., Melis, A. P., Hanus, D., and Tomasello, M. (2007). Spontaneous altruism by chimpanzees and young children. PLoS Biol. 5, e184. doi:10.1371/journal.pbio.0050184

Wellman, H. M., and Liu, D. (2004). Scaling of theory-of-mind tasks. Child Dev. 75, 523-541.

Zahn-Waxler, C., Radke-Yarrow, M., Wagner, E., and Chapman, M. (1992). Development of concern for others. Dev. Psychol. 28, 126-136.

Conflict of Interest Statement: The authors declare that the research was conducted in the absence of any commercial or financial relationships that could be construed as a potential conflict of interest.

Received: 27 August 2011; accepted: 09 November 2011; published online: 19 December 2011.

Citation: Robbins E and Rochat P (2011) Emerging signs of strong reciprocity in human ontogeny. Front. Psychology 2:353. doi: 10.3389/fpsyg.2011.00353

This article was submitted to Frontiers in Developmental Psychology, a specialty of Frontiers in Psychology.

Copyright (c) 2011 Robbins and Rochat. This is an open-access article distributed under the terms of the Creative Commons Attribution Non Commercial License, which permits non-commercial use, distribution, and reproduction in other forums, provided the original authors and source are credited. 